Pixel sensitivity variations in a CdTe-Medipix2 detector using poly-energetic x-rays

This article has been downloaded from IOPscience. Please scroll down to see the full text article.

2011 JINST 6 C01059

(http://iopscience.iop.org/1748-0221/6/01/C01059)

View the table of contents for this issue, or go to the journal homepage for more

Download details:

IP Address: 137.138.124.233

The article was downloaded on 27/07/2012 at 08:33

Please note that terms and conditions apply. 


\title{
Pixel sensitivity variations in a CdTe-Medipix2 detector using poly-energetic x-rays
}

\section{R. Aamir, ${ }^{a}$ S.P. Lansley, ${ }^{a, b, 1}$ R. Zainon, ${ }^{a}$ M. Fiederle,,${ }^{c}$ A. Fauler,${ }^{c}$ D. Greiffenberg, ${ }^{c}$}

\author{
P.H. Butler ${ }^{a, d}$ and A.P.H. Butler ${ }^{d, e, f}$ \\ ${ }^{a}$ Dept. of Physics \& Astronomy, \\ University of Canterbury, Christchurch, New Zealand \\ ${ }^{b}$ The MacDiarmid Institute for Advanced Materials \& Nanotechnology, \\ University of Canterbury, Christchurch, New Zealand \\ ${ }^{c}$ Freiburger Materialforschungszentrum, Albert-Ludwigs-Universität, \\ Freiburg i. Br., Germany \\ ${ }^{d}$ European Centre for Nuclear Research (CERN), \\ Geneva, Switzerland \\ ${ }^{e}$ Dept. of Electrical \& Computer Engineering, \\ University of Canterbury, Christchurch, New Zealand \\ ${ }^{f}$ Department of Radiology, \\ University of Otago Christchurch, New Zealand \\ E-mail: stuart. lansley@canterbury.ac.nz
}

ABSTRACT: We have a 1-mm-thick cadmium telluride (CdTe) sensor bump-bonded to a Medipix2 readout chip. This detector has been characterized using a poly-energetic x-ray beam. Open beam images (i.e. without an attenuating specimen between the $\mathrm{x}$-ray source and the detector) have been acquired at room temperature using the MARS-CT system. Profiles of various rows and columns were analyzed for one hundred, 35-ms exposures taken with a bias voltage of -300 V (operating in electron collection mode). A region of increased sensitivity is observed around the edges of the detector. A reasonably periodic, repeatable variation in pixel sensitivity is observed. Some small regions with very low sensitivity and others with zero signals are also observed. Surrounding these regions are circular rings of pixels with higher counts. At higher flux (higher tube current in the $\mathrm{x}$-ray source) there is evidence of saturation of the detector assembly. In this paper we present our understanding of the origin of these features and demonstrate the improved image quality obtained after correcting for these variations.

KEYWORDS: Solid state detectors; X-ray detectors; Pixelated detectors and associated VLSI electronics; Hybrid detectors

\footnotetext{
${ }^{1}$ Corresponding author.
} 


\section{Contents}

1 Introduction 1

2 MARS-CT background 2

3 Experimental setup 2

4 Results 2

4.1 Flood image 2

4.2 Edge effect 3

4.3 Individual pixel sensitivity 3

4.4 Pixel sensitivity response of neighboring pixels 3

4.5 Dark frame sensitivity 5

$\begin{array}{lll}4.6 & \text { Saturation effect } & 6\end{array}$

4.7 Flat field correction $\quad 7$

$\begin{array}{lll}4.8 & \text { Demonstration of image correction } & 7\end{array}$

5 Summary and conclusions $\quad 8$

\section{Introduction}

The Medipix detectors are single-photon counting detectors which convert x-rays into electronhole pairs by the help of a semiconductor layer on top of a CMOS readout chip. These detectors are referred to as hybrid detectors because they have two layers connected via bump bonds [1]. A bias voltage is applied to the sensor to transfer charge to the readout chip.

In Medipix detectors, the sensor and readout chip usually have pixels of the same size. Thus a photon impinging on one pixel of the sensor will be counted by the corresponding pixel of the readout chip. Strictly speaking, as the sensor is not pixilated, its pixel size is only defined by the pitch of the metallization on the collecting side of the sensor. Further, due to the influence of charge sharing, very often the photon is registered in several pixels.

The choice of the semiconductor sensor material depends on the application. Silicon is used due to the availability of high quality homogeneous material with few defects and high transport properties of the charge carriers around $1 \mathrm{~cm}^{2} / \mathrm{V}$. However, for a 300- $\mu \mathrm{m}$-thick sensor, its $\mathrm{x}$-ray absorption efficiency is less than $30 \%$ for energies above $20 \mathrm{keV}$ and less than $5 \%$ above $40 \mathrm{keV}$ [2]; this is due to the low atomic number of $\mathrm{Si}(\mathrm{Z}=14)$. Other materials, such as GaAs and $\mathrm{CdTe}$, have also been realized and tested. These show good detection efficiency for energies up to 80 and $100 \mathrm{keV}$ respectively, making them suitable for medical imaging $[3,4]$.

CdTe sensors have been processed with pixel contacts and passivation, and bump-bonded to Medipix 2 readout chips. Open beam images have been acquired (i.e. without an attenuating specimen between the x-ray source and the detector) using the MARS-CT scanner system. Our aim 
is to analyze the behavior of the individual pixels $\left(55 \times 55 \mu \mathrm{m}^{2}\right)$ of the CdTe sensor as well as comparing their response to that of neighboring pixels, by using a broad spectrum x-ray source.

\section{MARS-CT background}

The MARS-CT scanner is a multi-energy (spectroscopic) x-ray CT scanner that uses CERN's Medipix x-ray detectors. The first version of a prototype spectroscopic CT scanner, 'MARS-1', was constructed in 2007 as a joint venture between the University of Canterbury, Canterbury District Health Board and the University of Otago [5]. Images taken with the Medipix spectroscopic imaging detector have simultaneous spatial, energy and temporal resolution, so the CT system is referred to as Medipix All Resolution System CT (MARS-CT). Initial results proved the feasibility of a scanner that would take full advantage of the high spatial resolution that Medipix offers and would utilize most of the energy information, with low charge sharing [6, 7]. In this work we look at the initial characterization of a CdTe-Medipix2 detector within a MARS-CT scanner.

\section{Experimental setup}

For this experiment we have been using a 1-mm-thick CdTe sensor bump-bonded to a Medipix2 readout chip, operating at a $-300 \mathrm{~V}$ bias voltage in electron collection mode. The CdTe material was purchased from Acrorad [8] and the detector was bump-bonded at the FMF, Freiburg. The detector was flood irradiated using a 0.5 -mm-Al-filtered poly-energetic x-ray source, Thermo scientific Kevex PXST-150-75, operating at $75 \mathrm{kV}$ with a tube current of $150 \mu \mathrm{A}$ and a W target; the focal spot of the x-rays was $\sim 40 \mu \mathrm{m}$. The source to detector distance was $115 \mathrm{~mm}$. The readout was done using MUROS2 [9]. Before the measurements, a threshold equalization was performed using Pixelman software with respect to the noise edge [10].

A hundred flood frames were obtained with the low threshold set to $12 \mathrm{keV}$ and an exposure time of each acquisition of $35 \mathrm{~ms}$; the total scanning time was around one minute, over which time the x-ray output is measured to be stable. The uniformity of individual pixels and their response with respect to their neighboring pixels have been analyzed.

\section{Results}

\subsection{Flood image}

In order to check the sensitivity of CdTe sensor, one hundred flood images were obtained using the parameters described above. A typical image is shown in figure 1.

The mean count rate of the detector is $3073 \pm 393$ counts/pixel across the hundred frames. A region with increased sensitivity is observed at the edges of the detector. There are clusters of pixels with zero counts, a total of 112 pixels of the 65,536. There are also low and high sensitive pixels within the range of 2-2500 and 3500-6000 counts/pixel respectively. Importantly, around low sensitivity pixels, rings of pixels with higher photon counts are observed. The wrinkle pattern shows some inhomogeneities in the CdTe sensor. One of our goals in characterizing the CdTe Medipix 2 is to be able to correct for these imperfections. 


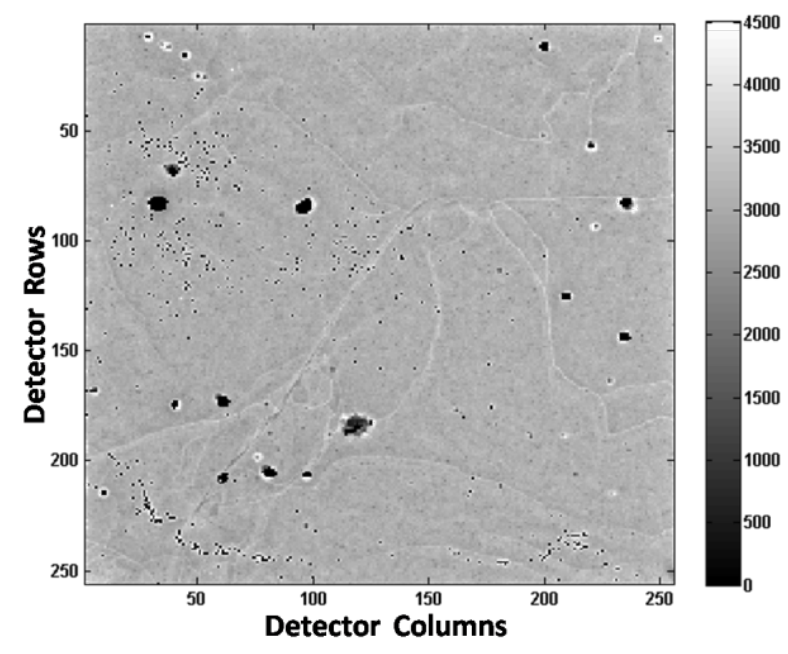

Figure 1. A flood image taken with CdTe, showing inhomogeneity of the detector.

\subsection{Edge effect}

A single frame (figure 2(a)) has been analyzed to see the effect that high pixel counts around the edge of detector have on the mean number of counts across rows (figure 2(b)) and columns (figure 2(c)). Mean counts of each row and column are plotted.

A few rows of pixels near the top edge of the detector (figure 2(a)) have a high mean number of counts (top of figure 2(b)). Across most of the detector the mean number of counts along a row lies close to the mean for the whole detector (within $\pm 2 \%$ of the detector mean, as shown by the dashed lines). Mean row values more than $2 \%$ below the detector mean arise from the low sensitivity pixels (discussed later in 4.3) in a row. A greater concentration of these pixels towards the bottom of the detector is responsible for the general decrease in mean number of counts. A few rows at the bottom edge of the detector also show higher mean counts. A similar response is observed for the mean values of individual columns (figure 2(c)).

The high counts observed at the detector edges are due to the volume effect, where the distance between the outermost pixel bump bonds and the edge of the sensor is greater than the pixel pitch. Figure 2(c) shows that the right hand side of the detector had more columns lying outside this limit.

\subsection{Individual pixel sensitivity}

The pixels have been divided into three categories: high sensitivity pixels (HSP), with counts $\geqslant 110 \%$ of the mean; low sensitivity pixels (LSP), with counts $\leqslant 90 \%$ of the mean; and average or normal pixels, with counts between $90 \%$ and $110 \%$ of the mean. Figure 3 shows all pixels by masking them according to these three categories.

\subsection{Pixel sensitivity response of neighboring pixels}

Several rows and columns of pixels have been analyzed across all frames to understand the sensitivity response of individual pixels. Figure 4(a) shows the profile of column [114] across 100 frames. The LSPs near rows 97 to 105 do not have a zero counting pixel nearby, but column [114] goes through a cluster of zero counting pixels at rows 185-190. Observe the quasi-periodic oscillation 

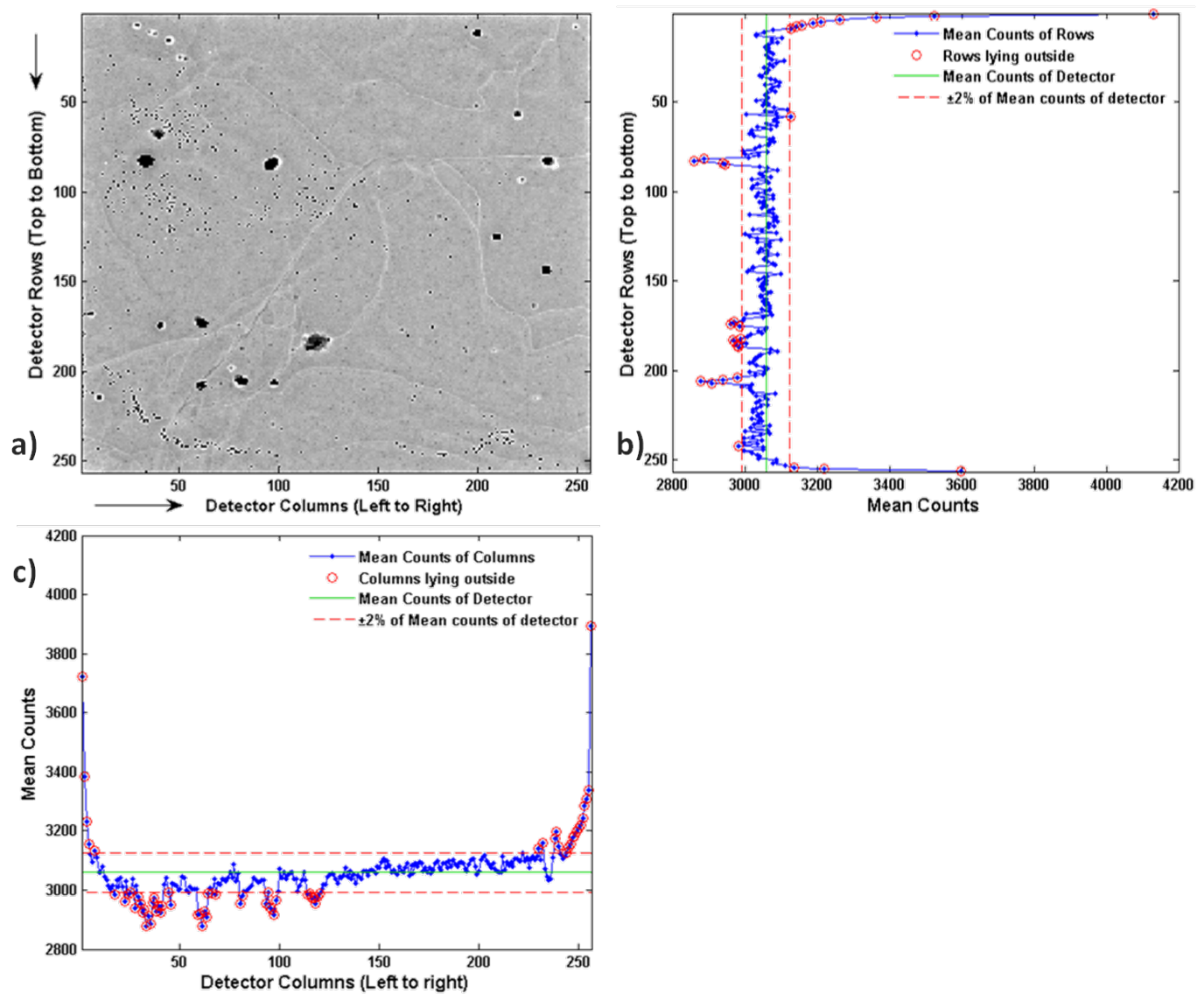

Figure 2. (a) a single frame, and the mean pixel counts for (b) rows, from top to bottom, and (c) columns, from left to right.

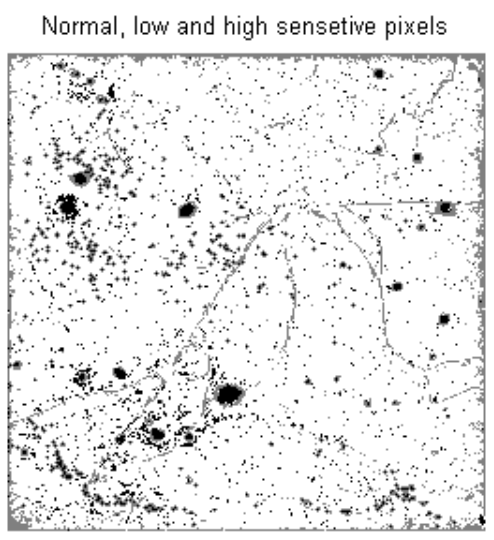

Figure 3. Normal, high and low sensitive pixels are shown by white, gray and black regions respectively.

in sensitivity around the 5 pixel $(250 \mu \mathrm{m})$ scale. Figure $4(\mathrm{~b})$ shows the high degree of repeatability across one hundred exposures.

We observed that in the vicinity of the low sensitive pixels there is a complete or partial ring of increased sensitivity. Figure 5(b) shows a typical region of the detector where such inhomogeneities 

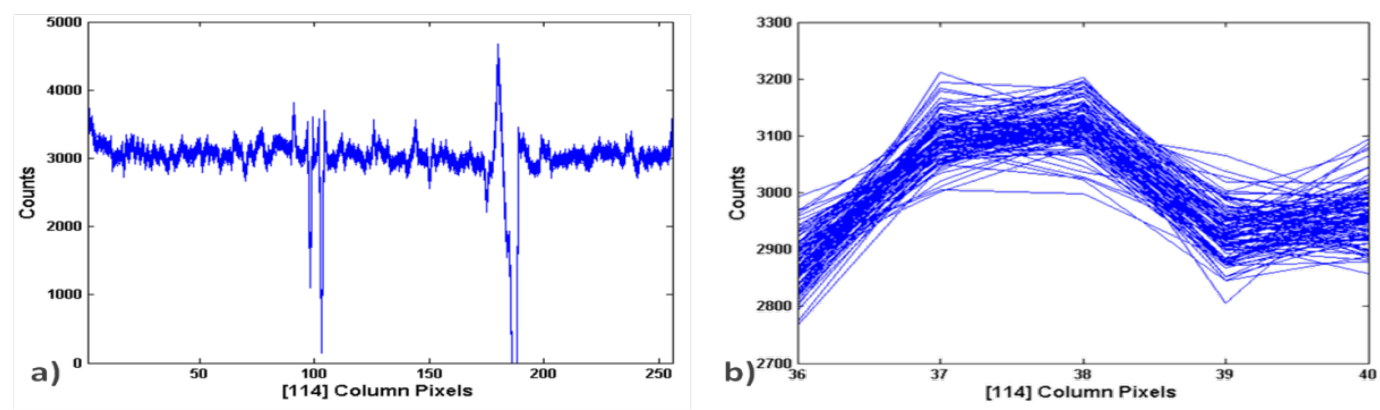

Figure 4. (a) Profile of 256 pixels of column [114], with 100 exposures superimposed. (b) Zoom in view of few pixels in (a). Each line indicates the measured counts for individual pixels in a single frame.
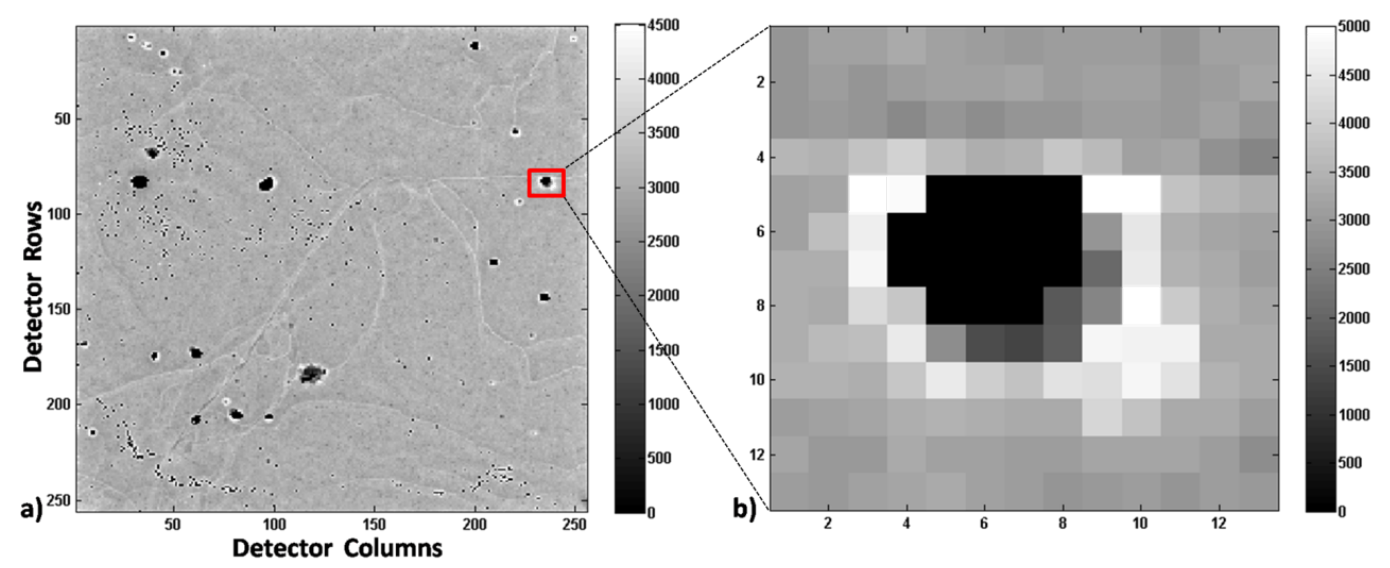

Figure 5. (a) Flood image of entire detector. (b) A region of 13 x 13 pixels selected from inhomogeneous region as marked in (a). Rings of pixels with higher photon counts are clearly observed.

are observed; the location of this region on the full image is shown in figure 5(a). It is observed that high sensitivity pixels form a ring around the low sensitivity pixels. Various reasons could account for this type of detector inhomogeneity.

\subsection{Dark frame sensitivity}

The dark response of pixels was investigated by acquiring one hundred frames using the same parameters as for the flood images but with the x-ray source off. A few pixels are observed to give non-zero counts even with no photons hitting the detector.

To analyze this response, matrices of $10 \times 10$ pixels containing such dark-counting pixels in them have been taken from a dark frame and compared with the same detector region from a flood image. Figure 6 shows a 3-D comparison of one particular region from (a) a dark frame and (b) a flood image. Three pixels from the dark frame show a dark-counting behavior. The same pixels in the flood frame show a low sensitivity response with respect to mean signal. Overall in both frames (dark and flood), these pixels produce equal counts and it seems that they are not responding to x-rays. This behavior in dark frames was observed only in small number of pixels in the form of clusters with sizes from two pixels to seven pixels. It is believed that this relates to the electronic noise in the Medipix ASIC. 

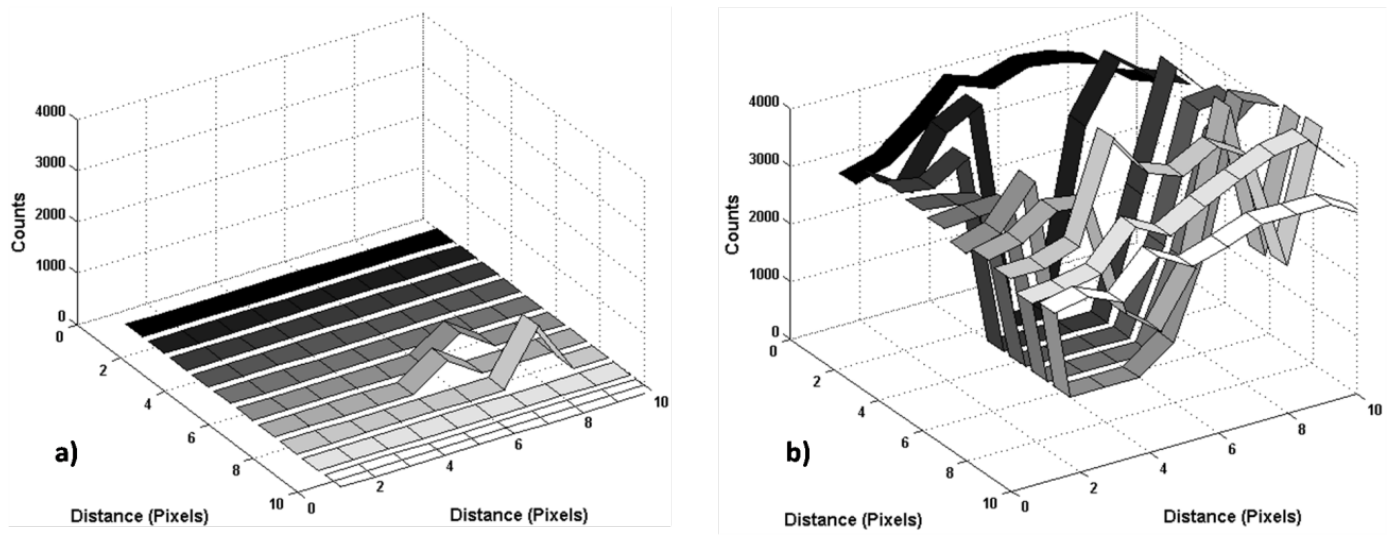

Figure 6. Three dimensional matrices of $10 \times 10$ pixels from (a) dark frame and (b) flood image. Three pixels showing a response in dark frame behave as low sensitive pixels in the flood exposure.
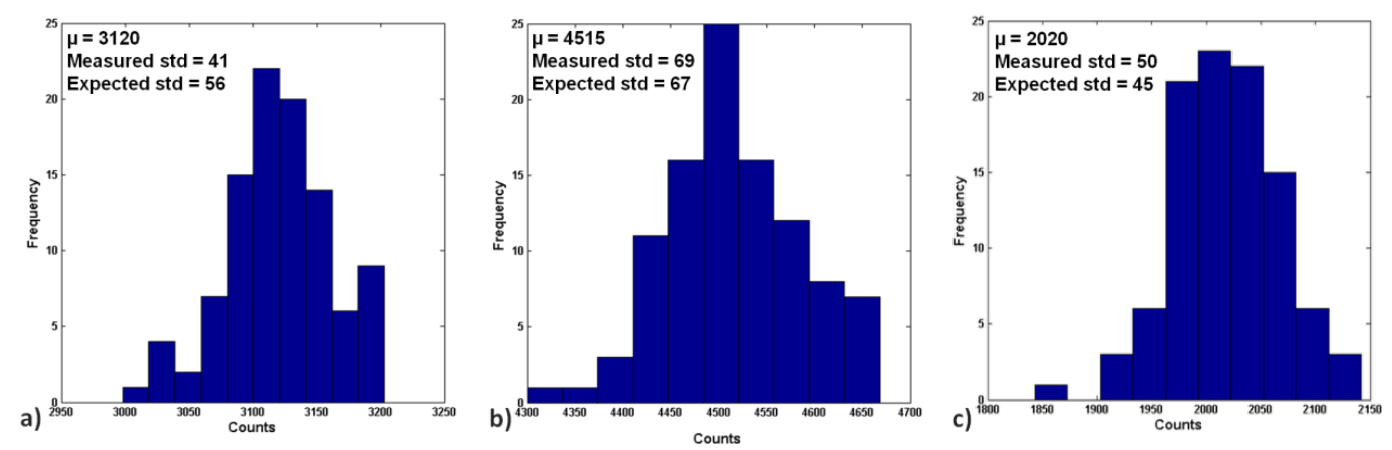

Figure 7. Histogram of the count values of (a) an average pixel $=[38 ; 114]$, (b) a high sensitive pixel $=$ $[180 ; 114]$ and (c) a low sensitive pixel $=[183 ; 114]$ across a hundred 35-ms, $150 \mu \mathrm{A}$ exposures.

\subsection{Saturation effect}

In figure 4(b) the individual counts in 5 pixels was shown over one hundred frames. Count histograms of an average pixel, a HSP and a LSP from column [114] are shown in figure 7(a), (b) and (c), respectively. For a purely Poisson distribution with no additional noise, the standard deviation is the square root of the number of counts. Almost all (98\%) of the pixels lie well below the Poisson noise limit. HSPs in the vicinity of low sensitive regions are close to the Poisson noise but LSPs are noisier.

To demonstrate this, a series of a hundred flood images were acquired at different $\mathrm{x}$-ray tube currents, keeping the tube voltage at $75 \mathrm{kVp}$. The low threshold was set to $12 \mathrm{keV}$ and the exposure time for each acquisition was $35 \mathrm{~ms}$, as for the previous flood images.

Figure 8 shows that at the lower flux levels, i.e. lower tube currents, the detector measures the photon beam with Poisson-distributed counts. As the photon flux (tube current) is increased, the ratio of measured noise to expected noise decreases to $80 \%$. This could be due to pulse pile up in the Medipix pixel electronics, or due to the saturation effect of sensor at high flux rates. Pulse pile up will occur well below the maximum counting rate of the Medipix pixels, because we only count a photon if there a pulse above our threshold of $12 \mathrm{keV}$, but with charge sharing, there will be many 


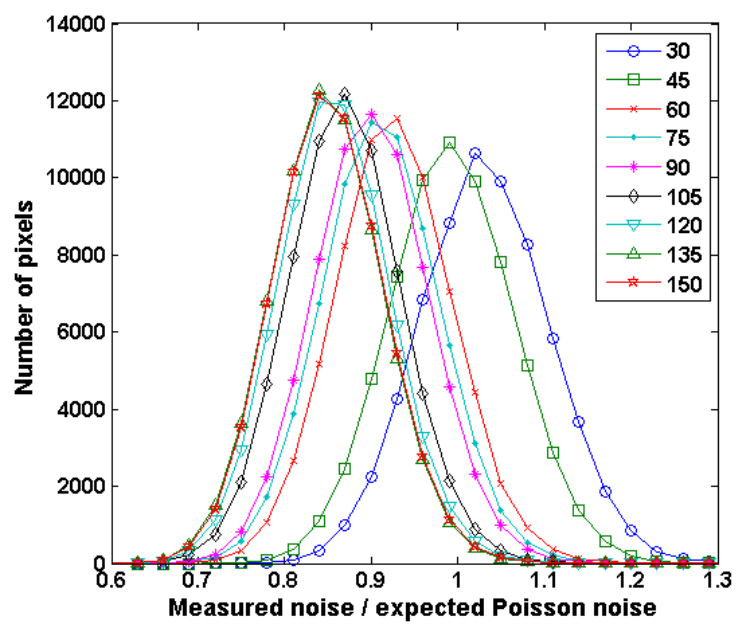

Figure 8. Ratio of measured noise to expected noise; tube currents are shown in $\mu \mathrm{A}$. The ratio decreases with increasing in tube current.

pulses below threshold. The reason for saturation of the sensor could be the scattering of charge carriers at high photon fluxes. This scattering phenomenon would be more predominant due to the compound nature of the sensor material (CdTe), which introduces inhomogeneities in the sensor due to grain boundaries, surface defects, etc. [11]. These disturb the electric field distribution and affect the charge collection efficiency of the pixels.

\subsection{Flat field correction}

Having measured and characterised inhomogeneties in the CdTe sensor we now wish to correct for them. This procedure is sometimes called a flat field correction or gain correction. The correction map is obtained by taking the ratio of the median counts over one hundred frames for each pixel to the median counts averaged over the whole detector. We use median rather than mean to remove outliers. The correction map consists of a coefficient for each individual pixel. By dividing the raw data by this correction map, counts for higher in the sensitivity pixels are reduced while the counts in the lower sensitivity pixels are increased. There are some regions where pixel sensitivity is so low that correction is difficult. Figure 9 shows the profile across column [114] for one hundred frames before and after this flat field correction.

\subsection{Demonstration of image correction}

We now apply this map to an imaging example. Two hundred images of a mobile phone SIM card were acquired by placing the SIM card between the x-ray source and the detector. The average image was corrected by generating a correction map from an average image of two hundred open beam images. A lower photon flux (tube current of $75 \mu \mathrm{A}$ ) was used to reduce saturation of the detector; all other parameters were as previously described. Figure 10 shows (a) the average open beam image, (b) the average image of the SIM card, and (c) the corrected image of the SIM card. The corrected image shows a clear improvement with the wrinkle pattern removed. 

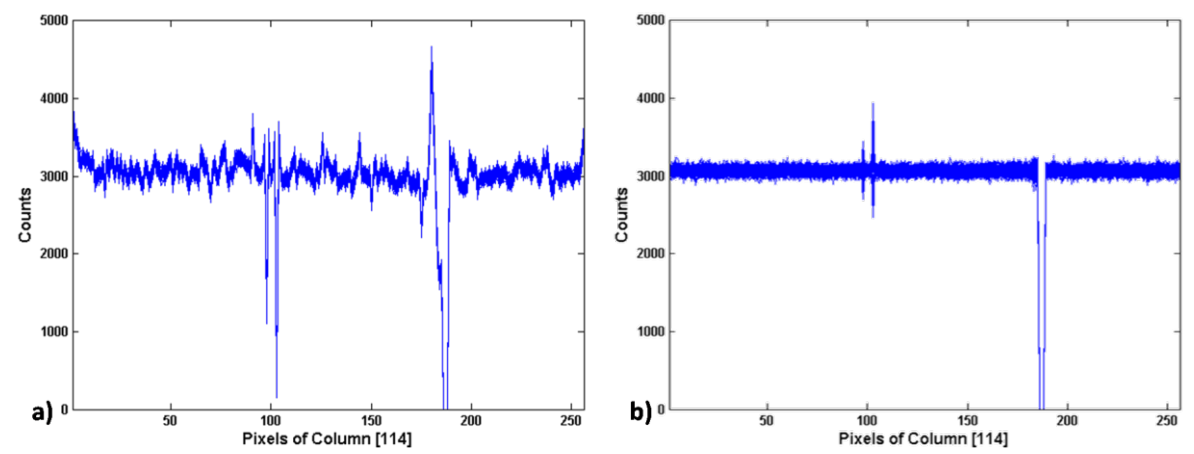

Figure 9. Column [114] profile of the detector (a) before calibration, and (b) after sensor calibration.
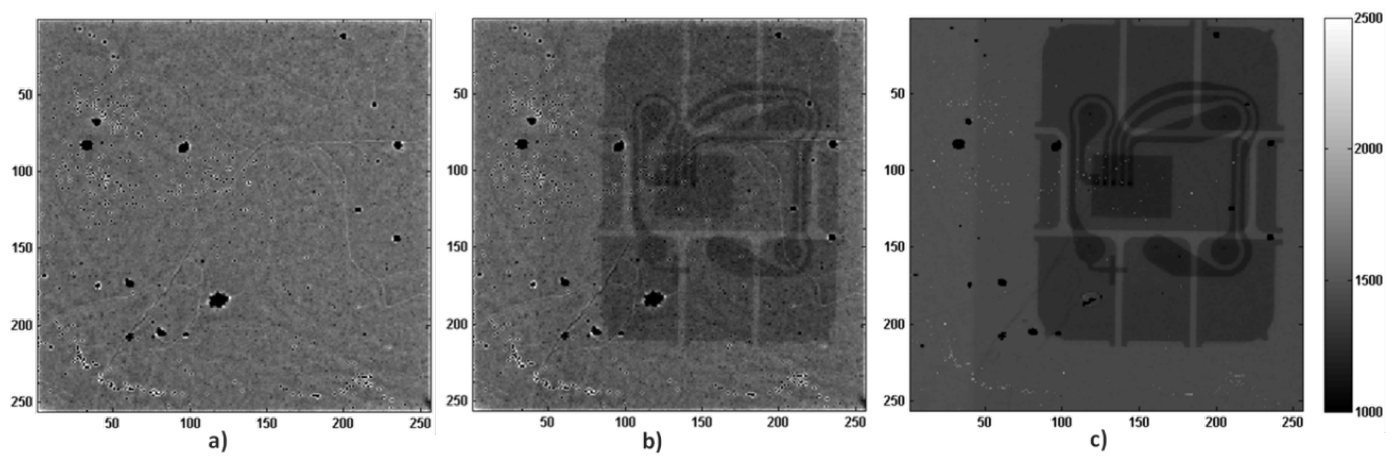

Figure 10. (a) Average of two hundred open beam frames, (b) average of two hundred frames of SIM card and (c) corrected image. The corrected image is the pure flat field image without further data processing (masking or interpolation on dead pixels). The gray scale has been set so that the images can be differentiated well. The flood frames were acquired just after the SIM card image.

\section{Summary and conclusions}

In this experimental study, we have characterized a 1-mm-thick CdTe sensor bump bonded to a Medipix 2 chip. We have analyzed the sensitivity of 65,536 pixels over a hundred frames: We have shown a high degree of repeatability across one hundred consecutive exposures. With the detector tested, only $0.04 \%$ of the pixels are dead, giving zero counts. We have observed a saturation effect at higher flux rates. We have performed a flat field correction and have demonstrated the improvement this has on image quality. However, dead regions still remain.

The future plan is to use CdTe-Medipix assemblies for computer tomography, where a volumetric reconstruction is obtained from a large number of projection images taken at different angles (approximately one image per degree). Correction strategies are therefore based on the requirements of the reconstruction technique. For filtered back projection a complete projection without missing data is generally required. In this case it is possible to interpolate the missing data, e.g. by linear interpolation across the dead region. Real data of the object is then obtained with the detector at a different gantry angle or bed location. While the interpolation will introduce some noise or artifacts, the large number of projections minimizes these. For algebraic reconstruction techniques missing data from dead regions represents rays with no information. These few rays are simply removed from the large set of simultaneous equations. 


\section{Acknowledgments}

This work was funded by Foundation for Research Science and Technology, NZ. The authors would like to thank all members of MARS-CT project, the Medipix 2 collaboration, and the Medipix 3 collaboration.

\section{References}

[1] B. Mikulec, Development of segmented semiconductor arrays for quantum imaging, Nucl. Instrum. Meth. A 510 (2003) 1.

[2] B. Mikulec, Single photon detection with semiconductor pixel arrays for medical imaging applications, Ph.D. Thesis, University of Vienna, Austria (2000), http://cdsweb.cern.ch/record/449988/files/thesis-2000-021.pdf.

[3] O. Limousin, New trends in CdTe and CdZnTe detectors for X-and gamma-ray applications, Nucl. Instrum. Meth. A 504 (2003) 24.

[4] A. Zwerger et al., Medipix2: Processing and measurements of GaAs pixel detectors, Nucl. Instrum. Meth. A 576 (2007) 23.

[5] P.H. Butler et al. Applying CERN's detector technology to health: MARS Biomedical 3D spectroscopic $x$-ray imaging, talk given at International Symposium on Peaceful Applications of Nuclear Technologies in the GCC Countries, April 14-16, Jeddah, Saudi Arabia (2008), http://ir.canterbury.ac.nz/bitstream/10092/2876/1/12611422_Butler_2008_Saudi.pdf.

[6] L. Tlustos, Spectroscopic X-ray imaging with photon counting pixel detectors, Nucl. Instrum. Meth. A 623 (2010) 823.

[7] A.P.H. Butler et al., Bio-medical X-ray imaging with spectroscopic pixel detectors, Nucl. Instrum. Meth. A 591 (2008) 141.

[8] Acrorad, http://www.acrorad.co.jp/us/index.html

[9] D. San Segundo Bello et al., An interface board for the control and data acquisition of the Medipix2 chip, Nucl. Instrum. Meth. A 509 (2003) 164.

[10] T. Holy et al., Data acquisition and processing software package for Medipix2, Nucl. Instrum. Meth. A 563 (2006) 254.

[11] G. Lutz, Semiconductor radiation detectors, Springer, U.S.A. (1999). 